\title{
WEIGHTED NORM INEQUALITIES FOR SINGULAR AND FRACTIONAL INTEGRALS
}

BY

\author{
BENJAMIN MUCKENHOUPT( $\left.{ }^{1}\right)$ AND RICHARD L. WHEEDEN $\left({ }^{2}\right)$
}

\begin{abstract}
Inequalities of the form $\left\||x|^{\alpha} T f\right\|_{q} \leqq C\left\||x|^{\alpha} f\right\|_{p}$ are proved for certain well-known integral transforms, $T$, in $E^{n}$. The transforms considered include Calderón-Zygmund singular integrals, singular integrals with variable kernel, fractional integrals and fractional integrals with variable kernel.
\end{abstract}

1. Introduction. We are interested in proving inequalities of the form

$$
\left\||x|^{\alpha} T f\right\|_{q} \leqq C\left\||x|^{\alpha} f\right\|_{p}
$$

Suppose for example that $T$ is a Calderón-Zygmund singular integral operator

$$
(T f)(x)=\lim _{\varepsilon \rightarrow 0} \int_{|x-y|>\varepsilon} f(y) \frac{\Omega(x-y)}{|x-y|^{n}} d y .
$$

It is by now a familiar fact (see [4]) that if $f(x) \in L^{p}\left(E^{n}\right), 1<p<\infty$, and $\Omega(x)$ is positively homogeneous of degree zero with mean value zero on the unit sphere, $\Sigma$, and

$$
N_{1}=1+\int_{\Sigma}\left(|\Omega(x)-\Omega(-x)|+|\Omega(x)+\Omega(-x)| \log ^{+}|\Omega(x)+\Omega(-x)|\right) d x^{\prime}
$$

is finite where $d x^{\prime}$ is the element of area on $\Sigma$, then (1.1) holds for $\alpha=0$ and $q=p$. The effort to obtain a similar result for other values of $\alpha$ has a long history. The case $n=1$ was studied originally by Hardy and Littlewood [5] and later by Babenko [1], who showed the result is true for $-1 / p<\alpha<1 / p^{\prime}$ where $1 / p+1 / p^{\prime}=1$. For $n>1$, Krée [6] used a rotation method to show the same is true under the above assumptions on $\alpha$.

For $n>1$, however, simple examples show some additional hypotheses on $\Omega$ are necessary to enlarge the $\alpha$ range. Let

$$
N_{r}=\left[\int_{\Sigma}|\Omega(x)|^{r} d x^{\prime}\right]^{1 / r}
$$

Received by the editors June 5, 1970 and, in revised form, February 4, 1971.

AMS 1969 subject classifications. Primary 4430, 4450.

Key words and phrases. Singular integrals, fractional integrals, weighted norm inequalities.

( $\left.{ }^{1}\right)$ Supported in part by NSF Grant GP-11403.

(2) Supported in part by NSF Grant GP-8556.

Copyright (C) 1971, American Mathematical Society 
for $r>1$ with the usual convention when $r=\infty$. Under the assumption that $N_{\infty}<\infty$, Stein [9] obtained the range $-n / p<\alpha<n / p^{\prime}$. Strichartz [10] proved (1.1) for $-n / p<\alpha<n / p^{\prime}$ provided that $r^{\prime} \leqq p \leqq r, 2(n-1)<r<\infty$ and $N_{r}<\infty$. Finally, using rather general methods Walsh [11] further improved the result, obtaining $\max \left(-n / p,-n / r^{\prime}\right)<\alpha<\min \left(n / p^{\prime}, n / r^{\prime}\right)$ provided $r>1$ and $N_{r}<\infty$.

Each of these results is included as a special case in the following theorem.

THEOREM 1. Suppose that $1<p<\infty, \Omega$ is homogeneous of degree 0 and has mean value 0 over $\Sigma$, and $r \geqq 1$. Then there is a constant, $C$, independent of $f$ and $\Omega$ such that

$$
\left\||x|^{\alpha} \sup _{\varepsilon>0}\left|\int_{|y|>\varepsilon} \frac{\Omega(y)}{|y|^{n}} f(x-y) d y\right|\right\|_{p} \leqq C N_{r}\left\||x|^{\alpha} f(x)\right\|_{p}
$$

provided $\max \left(-n / p,-1 / p-(n-1) / r^{\prime}\right)<\alpha<\min \left(n / p^{\prime}, 1 / p^{\prime}+(n-1) / r^{\prime}\right)$. There is no such $C$ if $\alpha \leqq-n / p, \alpha<-1 / p-(n-1) / r^{\prime}, \alpha \geqq n / p^{\prime}$ or $\alpha>1 / p^{\prime}+(n-1) / r^{\prime}$.

The inequality in Theorem 1 could be obtained from the results of Walsh, Krée, and Calderón and Zygmund by splitting up the integrals and using an interpolation argument. The virtue of the method used here is that it is direct and simple, using only the Calderón-Zygmund result, Hölder's inequality and Minkowski's integral inequality. Another advantage is that the procedure can be used to prove the following theorems.

THEOREM 2. Assume that $0<\gamma<n, 1<p<n / \gamma, 1 / q=1 / p-\gamma / n, r \geqq n /(n-\gamma)$, and $\Omega$ is homogeneous of degree 0 . Then there is a constant, $C$, independent of $f$ and $\Omega$ such that

$$
\left\||x|^{\alpha} \int_{E^{n}} \frac{\Omega(y)}{|y|^{n-\gamma}} f(x-y) d y\right\|_{\alpha} \leqq C N_{r}\left\||x|^{\alpha} f(x)\right\|_{p}
$$

provided that

$$
\gamma+\max \left(-n / p,-1 / p-(n-1) / r^{\prime}\right)<\alpha<-\gamma+\min \left(n / q^{\prime}, 1 / q^{\prime}+(n-1) / r^{\prime}\right) .
$$

There is no such $C$ if $\alpha \leqq \gamma-n / p, \alpha<\gamma-1 / p-(n-1) / r^{\prime}, \alpha \geqq-\gamma+n / q^{\prime}$ or $\alpha>-\gamma+1 / q^{\prime}$ $+(n-1) / r^{\prime}$.

For the following "variable kernel" theorem $N_{r}$ will be defined for $r>1$ as

$$
N_{r}=\sup _{x}\left[\int_{\Sigma}|\Omega(x, y)|^{r} d y^{\prime}\right]^{1 / r}
$$

THEOREM 3. Assume that $1<p<\infty, r \geqq p^{\prime}, \Omega(x, y)$ is homogeneous of degree 0 in $y$ and $\int_{\Sigma} \Omega(x, y) d y^{\prime}=0$ for every $x$. Then there is a constant, $C$, independent of $f$ and $\Omega$ such that

$$
\left\||x|^{\alpha} \sup _{\varepsilon>0}\left|\int_{|y|>\varepsilon} \frac{\Omega(x, y)}{|y|^{n}} f(x-y) d y\right|\right\|_{p} \leqq C N_{r}\left\||x|^{\alpha} f(x)\right\|_{p}
$$

if and only if $-n / p<\alpha<n / p^{\prime}-(n-1) / r$. There is no such $C$ for any value of $\alpha$ if $r<p^{\prime}$. 
Two other closely related results concerning variable kernel fractional integrals and a transformation related to the derivatives of the commutator of a singular integral operator are discussed briefly in $\$ 4$.

The method we use to prove the inequalities in Theorems 1-3 is somewhat standard. We split the integral defining $T$ into three parts. One of these contains the singularity of the kernel and can be handled by appealing to the known results on singular or fractional integrals. This part poses no restrictions on $\alpha$. The remaining two parts are estimated by techniques on the level of Hardy's inequality and impose the restrictions on $\alpha . \S \S 2$ and 3 are devoted to these estimates. $\S 4$ contains the proofs of the inequalities in Theorems 1-3 and remarks about applying this method to other transforms. $\$ 5$ contains examples to prove the negative assertions in Theorems 1-3. Whether or not the inequalities in Theorems 1 and 2 hold for the values of $\alpha$ for which no assertion is made seems to be a harder problem and is not treated here.

2. Simple lemmas. Throughout this paper, $x, y$ and $z$ will denote points in $E^{n}$ with projections $x^{\prime}=x /|x|, y^{\prime}=y /|y|$ and $z^{\prime}=z /|z|$ on the unit sphere, $\Sigma$. Integrals will be taken over $E^{n}$ or parts of $E^{n}$ unless specified to be over $\Sigma$ or parts of the real line. $\Omega(x, y)$ will denote a function homogeneous of degree 0 in $y$ and integrable on $\Sigma$ in $y$; for $r \geqq 1, \Omega_{r}$ will denote $\sup _{x}\left[\int_{\Sigma}|\Omega(x, y)|^{r} d y^{\prime}\right]^{1 / r}$ with the usual convention when $r=\infty . \Omega_{r}$ is the same as $N_{r}$ except for $r=1$; this notation is used to emphasize the fact that for the lemmas of $\$ \S 2$ and 3 the complicated expression, $N_{1}$, is not needed. If $\Omega(x, y)$ is independent of $x$, it will be written $\Omega(y)$. $C$ will denote a constant independent of the functions $f$ and $\Omega$ but not necessarily the same at each occurrence.

LEMMA 1. If $a>0,1 \leqq r \leqq \infty, 0<d \leqq r$ and $-n / d+(n-1) / r<b<\infty$, then

$$
\left[\left.\left.\int_{|y| \leqq a|x|}|| y\right|^{b} \Omega(x, x-y)\right|^{d} d y\right]^{1 / d} \leqq C|x|^{b+n / d} \Omega_{r}
$$

If $d=\infty$, then $r=\infty$ and the conclusion is obvious. If $d<\infty$, a change of variables shows that the $d$ th power of the left side of the conclusion equals

$$
\int_{|x-z| \leqq a|x|}|| x-\left.\left.z\right|^{b} \Omega(x, z)\right|^{d} d z .
$$

In this integral $|z| \leqq|x-z|+|x| \leqq(a+1)|x|$ so that $1 \leqq C(|x| /|z|)^{n-1}$. Therefore, (2.1) is bounded above by

$$
C|x|^{n-1} \int_{|z| \leqq(a+1)|x|}|| x-\left.\left.z\right|^{b} \Omega(x, z)\right|^{d}|z|^{1-n} d z .
$$

Changing to polar coordinates with $z=s z^{\prime}$ shows that (2.2) equals

$$
C|x|^{n-1} \int_{\Sigma}\left|\Omega\left(x, z^{\prime}\right)\right|^{d}\left[\int_{0}^{(a+1)|x|}\left|x-s z^{\prime}\right|^{b d} d s\right] d z^{\prime}
$$


To estimate (2.3) it will first be shown that

$$
\begin{aligned}
\int_{0}^{(a+1)|x|}\left|x-s z^{\prime}\right|^{b d} d s & \leqq C|x|^{b d+1}\left(1+\left|x^{\prime}-z^{\prime}\right|^{b d+1}\right), & & b d \neq-1, \\
& \leqq C\left(1+\log ^{+} 1 /\left|x^{\prime}-z^{\prime}\right|\right), & & b d=-1 .
\end{aligned}
$$

To do this let $\theta$ be the angle between $x^{\prime}$ and $z^{\prime}$ and observe that by the law of cosines $\left|x-s z^{\prime}\right|=\left(|x|^{2}-2 s|x| \cos \theta+s^{2}\right)^{1 / 2}$. Using this, a trigonometric identity and the change of variables $s=|x| t$ shows that the left side of (2.4) equals

$$
|x|^{b d+1} \int_{0}^{a+1}\left[(1-t)^{2}+4 t \sin ^{2} \frac{1}{2} \theta\right]^{b d / 2} d t .
$$

Since $\left|\sin \frac{1}{2} \theta\right|$ has the same order of magnitude as $\left|x^{\prime}-z^{\prime}\right|,(2.4)$ follows.

Now use (2.4) in (2.3). Hölder's inequality with exponents $r / d$ and $r /(r-d)$ then shows that (2.3) is bounded by

$$
C|x|^{b d+n}\left(\Omega_{r}\right)^{d}\left[\int_{\Sigma}\left(1+\left|x^{\prime}-z^{\prime}\right|^{b d+1}\right)^{r /(r-d)} d z^{\prime}\right]^{(r-d) / r}
$$

if $b d \neq-1$ and

$$
C|x|^{b d+n}\left(\Omega_{r}\right)^{d}\left[\int_{\Sigma}\left(1+\log ^{+} 1 /\left|x^{\prime}-z^{\prime}\right|\right)^{r /(r-d)} d z^{\prime}\right]^{(r-d) / r}
$$

if $b d=-1$. The hypothesis on $b$ shows that $(b d+1) r /(r-d)>1-n$ so that the integral in (2.5) is bounded. Similarly, if $b d=-1, d<r$ so that $r /(r-d)<\infty$ and the integral in (2.6) is bounded. This completes the proof of Lemma 1.

LEMMA 2. If $a>0,1 \leqq r \leqq \infty, 0<d \leqq r$ and $b<-n / d$, then

$$
\left(\left.\left.\int_{|y| \geqq a|x|}|| y\right|^{b} \Omega(x, x-y)\right|^{d} d y\right)^{1 / d} \leqq C|x|^{b+n / d} \Omega_{r} .
$$

If $d=\infty$, then $r=\infty$ and the conclusion is obvious. If $d<\infty$, a change of variables shows that the $d$ th power of the left side of the conclusion equals

$$
\int_{|x-z| \geqq a|x|}|| x-\left.\left.z\right|^{b} \Omega(x, z)\right|^{d} d z
$$

In this integral $|x|+|z| \leqq 2|x|+|x-z| \leqq(1+2 / a)|x-z|$ and in any case $|x-z|$ $\leqq|x|+|z|$. Therefore $|x-z|$ can be replaced by $|x|+|z|$ to show that (2.7) is bounded above by

$$
C \int(|x|+|z|)^{b d}|\Omega(x, z)|^{d} d z .
$$

In polar coordinates $(2.8)$ becomes

$$
C \int_{\Sigma}\left|\Omega\left(x, z^{\prime}\right)\right|^{d}\left[\int_{0}^{\infty}(|x|+s)^{b d} s^{n-1} d s\right] d z^{\prime}
$$

which is bounded by the $d$ th power of the right side of the conclusion of Lemma 2 . 
The following forms of Minkowski's integral inequality will be needed, for $a>0$ and $1 \leqq s \leqq \infty$,

$$
\left[\int\left(\int_{|y| \geqq a|x|}|f(x, y)| d y\right)^{s} d x\right]^{1 / s} \leqq \int\left[\int_{|x| \leqq|y| /|a|}|f(x, y)|^{s} d x\right]^{1 / s} d y
$$

and

$$
\left[\int\left(\int_{|y| \leqq a|x|}|f(x, y)| d y\right)^{s} d x\right]^{1 / s} \leqq \int\left[\int_{|x| \geqq|y| / a}|f(x, y)|^{s} d x\right]^{1 / s} d y .
$$

3. Analogues of Hardy's inequality. In this section we will consider the following integrals for a fixed $a>0$,

$$
R=R(f, \Omega)=\int_{|y| \leqq a|x|} f(y) \Omega(x, x-y) d y
$$

and

$$
S=S(f, \Omega)=\int_{|y| \geqq a|x|} f(y) \Omega(x, x-y) d y .
$$

For $1 \leqq p \leqq \infty,\|\|_{p}$ denotes ordinary unweighted $L^{p}$ norm in $E^{n}$ and $p^{\prime}$ denotes the number such that $1 / p+1 / p^{\prime}=1$.

Lemma 3. If $\Omega(x, y)$ does not depend on $x, 0 \leqq \gamma \leqq n, 1 \leqq p \leqq n / \gamma, 1 / q=1 / p-\gamma / n$, $r \geqq n /(n-\gamma)$ and $\alpha<\min (-n / q,-1 / q-(n-1) / r)$, then

$$
\left\||x|^{\alpha} R\right\|_{q} \leqq C \Omega_{r}\left\||x|^{\alpha+n-\gamma} f\right\|_{p} .
$$

Let $m=0$ if $q=\infty$ and otherwise let $m=\min (1, r / q)$. Choose $\varepsilon$ so that $0<\varepsilon$ $<-\alpha-n / q+(n-1)(m-1) / r$. The hypothesis on $\alpha$ insures that such an $\varepsilon$ can be chosen. Using Hölder's inequality on the definition of $R$ shows that $|x|^{\alpha}|R|$ is bounded by the product of

$$
\left[\left.\left.\int_{|y| \leqq a|x|}|f(y)| y\right|^{\alpha+n-\gamma+\varepsilon}|\Omega(x-y)|^{m}\right|^{p} d y\right]^{1 / p}
$$

and

$$
|x|^{\alpha}\left[\int_{|y| \leqq a|x|}\left(|y|^{-\alpha-n+\gamma-\varepsilon}|\Omega(x-y)|^{1-m}\right)^{p^{\prime}} d y\right]^{1 / p^{\prime}} .
$$

If $m<1$, it is easy to verify that the exponents in (3.4) satisfy the hypotheses of Lemma 1 with $d=(1-m) p^{\prime}$ and $b=(-\alpha-n+\gamma-\varepsilon) /(1-m)$. Consequently, (3.4) is bounded by

$$
C|x|^{-8-n / q}\left(\Omega_{r}\right)^{1-m}
$$

If $m=1$, direct integration shows that (3.5) bounds (3.4). 
Using the fact that $|x|^{\alpha}|R|$ is bounded by the product of (3.3) and (3.5) shows that $\left\||x|^{\alpha} R\right\|_{q}$ is bounded by the product of $C\left(\Omega_{r}\right)^{1-m}$ and

$$
\left\|\left[\left.\left.\int_{|y| \leqq a|x|}|f(y)| y\right|^{\alpha+n-y+\varepsilon}|\Omega(x-y)|^{m}|x|^{-\varepsilon-n / q}\right|^{p} d y\right]^{1 / p}\right\|_{q} .
$$

If $q<\infty$, then $p<\infty$ and (2.10) shows that (3.6) is bounded above by

$$
\left(\int\left[\left.\left.\int_{|x| \geqq|y| / a}|| \Omega(x-y)\right|^{m}|x|^{-\varepsilon-n / q} f(y)|y|^{\alpha+n-y+\varepsilon}\right|^{q} d x\right]^{p / q} d y\right)^{1 / p} .
$$

Since $\Omega(-z)$ satisfies the same conditions as $\Omega(z)$, Lemma 2 with $d=m q$ and $b=(-\varepsilon-n / q) / m$ can be applied to the inner integral to show that (3.7) is bounded by

$$
C\left(\Omega_{r}\right)^{m}\left[\left.\left.\int|f(y)| y\right|^{\alpha+n-\gamma}\right|^{p} d y\right]^{1 / p}
$$

If $q=\infty$, then $m=0$ and it is easy to see directly that (3.6) is bounded by (3.8). The variable kernel version of Lemma 3 is the following.

LEMMA 4. If $0 \leqq \gamma \leqq n, 1 \leqq p \leqq n / \gamma, 1 / q=1 / p-\gamma / n, r \geqq p^{\prime}$ and $\alpha<-n / q-(n-1) / r$, then $\left\||x|^{\alpha} R\right\|_{q} \leqq C \Omega_{r}\left\||x|^{\alpha+n-\gamma} f\right\|_{p}$.

The proof is the same as for Lemma 3 replacing $m$ by 0. Expression (3.7) can be estimated directly in this case since the $\Omega$ drops out.

LEMMA 5. If $\Omega(x, y)$ does not depend on $x, 0 \leqq \gamma \leqq n, 1 \leqq p \leqq n / \gamma, 1 / q=1 / p-\gamma / n$, $r \geqq n /(n-\gamma)$ and $\alpha>\gamma+\max \left(-n / p,-1 / p-(n-1) / r^{\prime}\right)$, then

$$
\left\||x|^{\alpha} S\right\|_{q} \leqq C \Omega_{r}\left\||x|^{\alpha+n-\gamma} f\right\|_{p} .
$$

Let $g(x)$ be a function in $L^{q^{\prime}}$. Then $\int|x|^{\alpha} S g d x$ equals

$$
\int f(y)|y|^{\alpha+n-\gamma}\left[|y|^{-\alpha-n+\gamma} \int_{|x| \leqq|y| / a}|x|^{\alpha} g(x) \Omega(x-y) d x\right] d y .
$$

Now use Hölder's inequality on the outer integral with exponent $p$ on $f(y)|y|^{\alpha+n-\gamma}$ and exponent $p^{\prime}$ on the expression in brackets. Lemma 3 can then be applied to the second part to show that (3.9) is bounded by $\left\|f|y|^{\alpha+n-\gamma}\right\|_{p} \Omega_{r}\|g\|_{q^{\prime}}$. Since $g$ was an arbitrary function in $L^{q^{\prime}}$, this completes the proof of Lemma 5.

LEMMA 6. If $0 \leqq \gamma \leqq n, 1 \leqq p \leqq n / \gamma, 1 / q=1 / p-\gamma / n, r \geqq p^{\prime}$ and $\alpha>-n / q$, then $\left\||x|^{\alpha} S\right\|_{q} \leqq C \Omega_{r}\left\||x|^{\alpha+n-\gamma} f\right\|_{p}$.

Choose $\varepsilon$ so that $0<\varepsilon<\alpha+n / q$. Hölder's inequality shows that $|x|^{\alpha} S$ is bounded by the product of

$$
\left[\left.\left.\int_{|y| \geqq a|x|}|f(y)| y\right|^{\alpha+n-y-\varepsilon}\right|^{p} d y\right]^{1 / p}
$$

and

$$
|x|^{\alpha}\left[\left.\left.\int_{|y| \geqq a|x|}|| y\right|^{-\alpha-n+y+\varepsilon} \Omega(x, x-y)\right|^{p^{\prime}} d y\right]^{1 / p^{\prime}}
$$


Lemma 2 shows that

$$
\left.\left.|| x\right|^{\alpha} S\left|\leqq C \Omega_{r}\right| x\right|^{\varepsilon-n / q}\left[\left.\int_{|y| \geqq a|x|}|f(y)| y\right|^{\alpha+n-\gamma-\left.\varepsilon\right|^{p}} d y\right]^{1 / p} .
$$

Then use (3.12) to estimate $\left\||x|^{\alpha} S\right\|_{q}$ and apply (2.9). The conclusion of Lemma 6 follows immediately.

4. Proof of the inequalities in Theorems 1-3. The theorems will be proved simultaneously. Define

$$
T(x)=\sup _{\varepsilon>0}\left|\int_{|y|>\varepsilon} \frac{f(x-y) \Omega(x, y)}{|y|^{n-y}} d y\right|
$$

where $\Omega(x, y)=\Omega(y)$ in the case of Theorems 1 and 2 and $\gamma=0$ in the case of Theorems 1 and 3. Next, define $A_{k}$ to be the set of all $x$ with $2^{k-1} \leqq|x|<2^{k+2}, B_{k}$ the set of all $x$ with $2^{k} \leqq|x|<2^{k+1}$ and let $c_{k}(x)$ be the characteristic function of $B_{k}$. Then let

$$
T^{*}(x)=\sup _{\varepsilon>0}\left|\sum_{k=-\infty}^{\infty} c_{k}(x) \int_{y \in A_{k},|x-y|>\varepsilon} \frac{f(y) \Omega(x, x-y)}{|x-y|^{n-\gamma}} d y\right| .
$$

It follows immediately from (4.1) and (4.2) that

$$
\left|T(x)-T^{*}(x)\right| \leqq C\left[|x|^{\gamma-n} R(|f|,|\Omega|)+S(g,|\Omega|)\right]
$$

where $g(y)=|y|^{\gamma-n}|f(y)|$ and $R$ and $S$ are the operators defined in (3.1) and (3.2) with $a=1$. Using Lemmas 3-6 shows that

$$
\left\||x|^{\alpha}\left[T(x)-T^{*}(x)\right]\right\|_{q} \leqq C \Omega_{r}\left\||x|^{\alpha} f(x)\right\|_{p}
$$

for the stated values of $p, q, r$ and $\alpha ; q=p$ in the case of Theorems 1 and 3. Consequently, the proof of the inequalities can be completed by showing for the stated values of $p, q$ and $r$ and every $\alpha$ that

$$
\left\||x|^{\alpha} T^{*}(x)\right\|_{q} \leqq C N_{r}\left\||x|^{\alpha} f(x)\right\|_{p} .
$$

To prove (4.4), start with the fact that $\left[\left\||x|^{\alpha} T^{*}(x)\right\|_{q}\right]^{q}$ equals

$$
\left.\left.\sum_{k=-\infty}^{\infty} \int_{x \in B_{k}} \sup _{\delta>0}|| x\right|^{\alpha} \int_{y \in A_{k},|x-y|>\varepsilon} \frac{f(y) \Omega(x, x-y)}{|x-y|^{n-\gamma}} d y\right|^{q} d x
$$

By the definition of $B_{k}$ this is majorized by

$$
\sum_{k=-\infty}^{\infty} C 2^{k \alpha q} \int_{x \in B_{k}} \sup _{\varepsilon>0}\left|\int_{y \in A_{k},|x-y|>\varepsilon} \frac{f(y) \Omega(x, x-y)}{|x-y|^{n-\gamma}} d y\right|^{q} d x .
$$

This is bounded by

$$
\sum_{k=-\infty}^{\infty} C 2^{k \alpha q} N_{r}^{q}\left[\int_{x \in A_{k}}|f(x)|^{p} d x\right]^{q / p}
$$


for Theorems 1 and 3 this follows from results in Calderón and Zygmund [4]; and for Theorem 2 it follows from Theorem 9 of [7]. The dependence on $N_{r}$ is not stated explicitly in [4] or [7] but is implicit in the proofs; it also follows from the Banach-Steinhaus theorem. It is not necessary to assume that $f$ is in $L^{p}$ to use these theorems; if $|x|^{\alpha} f$ is in $L^{p}$ for some $\alpha$ then $f$ belongs to $L^{p}$ on every ring $A_{k}$, which is enough.

Now (4.6) is clearly bounded by

$$
C \sum_{k=-\infty}^{\infty} N_{r}^{q}\left[\left.\left.\int_{x \in A_{k}}|| x\right|^{\alpha} f(x)\right|^{p} d x\right]^{q / p}
$$

Since $q / p \geqq 1$, this is bounded by

$$
C N_{r}^{q}\left[\left.\left.\sum_{k=-\infty}^{\infty} \int_{x \in A_{k}}|| x\right|^{\alpha} f(x)\right|^{p} d x\right]^{q / p} .
$$

Since each $x$ is in at most three $A_{k}$ 's, (4.7) is bounded by

$$
C N_{r}^{q}\left[\left.\left.\int_{E^{n}}|| x\right|^{\alpha} f(x)\right|^{p} d x\right]^{q / p}
$$

This completes the proof of (4.4) and, therefore, completes the proof of the inequalities in Theorems 1-3.

Theorems 1-3 have the following logical complement.

TheOREM 4. Assume that $0<\gamma<n, 1<p<n / \gamma, \Omega(x, y)$ is homogeneous of degree 0 in $y, 1 / q=1 / p-\gamma / n$, and $r>p^{\prime}$. Then there is a constant, $C$, independent of $f$ and $\Omega$ such that

$$
\left\||x|^{\alpha} \int_{E^{n}} \frac{\Omega(x, y)}{|y|^{n-\gamma}} f(y) d y\right\|_{q} \leqq C N_{r}\left\||x|^{\alpha} f(x)\right\|_{p}
$$

if and only if $-n / q<\alpha<n / q^{\prime}-(n-1) / r-\gamma$. There is no such $C$ for any value of $\alpha$ if $r<p^{\prime}$.

The proof given in this section applies equally well to Theorem 4 except for the passage from (4.5) to (4.6). For this an argument similar to that for fixed kernel fractional integrals in Theorem 13 of [7] can be given. The negative parts can be treated in the same manner that they are in $\$ 5$ for Theorem 3.

The inequality in Theorem 1 is also true as stated for

$$
(T f)(x)=\sup _{\varepsilon>0}\left|\int_{|x-y|>\varepsilon}[a(x)-a(y)] f(y) \frac{\Omega(x-y)}{|x-y|^{n+1}} d y\right|
$$

where $a$ has first partial derivatives bounded by $M, f$ is in $L^{p}, 1<p<\infty$, and $\Omega$ is in $L \log ^{+} L$ on $\Sigma$ and is orthogonal over $\Sigma$ to polynomials of degree 1 . To see this, split the integral as usual. Since $|a(x)-a(y)| \leqq M|x-y|,\left|T f-T^{*} f\right|$ is majorized 
by $M\left[|x|^{-n} R(|f|,|\Omega|)(x)+S\left(|f| /|y|^{n},|\Omega|\right)(x)\right]$ as usual. $\left\||x|^{\alpha} T^{*}(x)\right\|_{q}$ is estimated by the same argument as before using the known fact that $\|T f\|_{p} \leqq C M\|f\|_{p}$; see [2] and [3].

5. Proof of the negative assertions in Theorems 1-3. Simple examples will be given to show that the inequalities in Theorems 1-3 do not hold for the indicated ranges of $\alpha$.

In Theorem 2 if $\alpha \geqq-\gamma+n / q^{\prime}$, choose $\Omega(y) \equiv 1$ and $f(y)$ the characteristic function of $|y| \leqq 1$. Then for $|x| \geqq 2$ the right side of the inequality

$$
|x|^{\alpha} \int_{E^{n}} f(y) \frac{\Omega(x-y)}{|x-y|^{n-y}} d y \geqq C|x|^{\alpha+\gamma-n} \int_{|y|<|x| / 2} f(y) \Omega(x-y) d y
$$

exceeds a constant times $|x|^{\alpha+\gamma-n}$ and, therefore, does not belong to $L^{q}(|x| \geqq 2)$ for $\alpha$ in this range.

If $\alpha \geqq-\gamma+1 / q^{\prime}+(n-1) / r^{\prime}$ in Theorem 2 , fix a unit vector, $w^{\prime}$, let $\Omega(y)$ $=\left|w^{\prime}-y^{\prime}\right|^{-t / r}$ for some $t<n-1$ and $f(y)$ be the characteristic function of $|y| \leqq 1$. Then for $|x| \geqq 2$ the left side of (5.1) exceeds a constant times

$$
|x|^{\alpha+\gamma-n} \int_{|y|<1} \Omega(x-y) d y .
$$

Let $D$ be the intersection of $|x| \geqq 2$ and the cylinder with radius $\frac{1}{2}$ and axis the ray emanating from the origin along $w^{\prime}$. For $x$ in $D$ there is a positive constant, $C$, such that

$$
\int_{|x-y|<1} \Omega(y) d y \geqq C \int_{|x|-C}^{|x|+C} s^{n-1} d s \int_{\left|w^{\prime}-y^{\prime}\right| \leqq C /|x|}\left|w^{\prime}-y^{\prime}\right|^{-t / r} d y^{\prime}
$$

A simple computation shows that this is bounded below by $C|x|^{t / r}$. It follows that the integral over $D$ of the $q$ th power of (5.2) exceeds a positive constant times $\int_{D}|x|^{(\alpha+\gamma-n+t / r) a} d x$; this is infinite if the exponent is greater than or equal to -1 . Since $t$ is an arbitrary number less than $n-1$, this integral can always be made infinite if $\alpha>-\gamma+1 / q^{\prime}+(n-1) / r^{\prime}$.

In Theorem 1 if $\alpha \geqq n / p^{\prime}$ or $\alpha>1 / p^{\prime}+(n-1) / r^{\prime}, \Omega$ cannot be chosen to be positive since it must satisfy the additional requirement $\int_{\Sigma} \Omega=0$. If $\alpha \geqq n / p^{\prime}$, take $\Omega$ to be 1 on the upper hemisphere of $\Sigma$ and -1 on the lower hemisphere. The same reasoning as used before for $|x| \geqq 2$ can be used if $|x| \geqq 2$ and $x_{n} \geqq 1$. If $\alpha>1 / p^{\prime}$ $+(n-1) / r^{\prime}$, take $\Omega$ to be an odd function equal to $\left|w^{\prime}-y^{\prime}\right|^{-t / r}$ in a neighborhood of $w^{\prime}$ and zero away from $w^{\prime}$ and $-w^{\prime}$ and argue as before.

The rest of the negative assertions in Theorems 1 and 2 can be obtained by a duality argument.

To show that the range of $\alpha$ is best possible in Theorem 3 is even easier. For the lower bound this is already true if $\Omega$ does not depend on $x$. If $\alpha \geqq n / p^{\prime}-(n-1) / r$ in Theorem 3 , let $f(y)$ be the characteristic function of $|y| \leqq 1$ and $\Omega(x, y)$ be the 
odd function of $y$ equal to $|x|^{(n-1) / r}$ for $\left|x^{\prime}-y^{\prime}\right|<1 /|x|$ and zero away from $x^{\prime}$ and $-x^{\prime}$. Then $N_{r}<\infty$ and for $|x| \geqq 2$

$$
|x|^{\alpha} \int \frac{f(y) \Omega(x, x-y)}{|x-y|^{n}} d y \geqq C|x|^{\alpha-n} \int_{|x-y|<1} \Omega(x, y) d y,
$$

and this is bounded below by $C|x|^{\alpha-n+(n-1) / r}$. This is not in $L^{p}(|x| \geqq 2)$ for $\alpha$ in the given range.

If $r<p^{\prime}$, Theorem 3 is clearly false since the integral in $y$ on the left side of the conclusion may be infinite for every $x$ while the right side of the conclusion is finite.

\section{REFERENCES}

1. K. I. Babenko, On conjugate functions, Dokl. Akad. Nauk SSSR 62 (1948), 157-160. MR 10, 249.

2. B. Bajšanski and R. Coifman, On singular integrals, Proc. Sympos. Pure Math., vol. 10, Amer. Math. Soc., Providence, R. I., 1967, pp. 1-17. MR 38 \#6405.

3. A. P. Calderón, Commutators of singular integral operators, Proc. Nat. Acad. Sci. U.S.A. 53 (1965), 1092-1099. MR 31 \#1575.

4. A. P. Calderón and A. Zygmund, On singular integrals, Amer. J. Math. 78 (1956), 289-309. MR 18, 894.

5. G. H. Hardy and J. E. Littlewood, Some more theorems concerning Fourier series and Fourier power series, Duke Math. J. 2 (1936), 354-382.

6. P. Krée, Sur les multiplicateurs dans $F L^{p}$ avec poids, Ann. Inst. Fourier (Grenoble) 16 (1966), fasc. 2, 91-121. MR 35 \#7080.

7. B. Muckenhoupt and E. M. Stein, Classical expansions and their relation to conjugate harmonic functions, Trans. Amer. Math. Soc. 118 (1965), 17-92. MR 33 \#7779.

8. R. O'Neil, Convolution operators and L( $p, q)$ spaces, Duke Math. J. 30 (1963), 129-142. MR 26 \#4193.

9. E. M. Stein, Note on singular integrals, Proc. Amer. Math. Soc. 8 (1957), 250-254. MR 19 547.

10. R. S. Strichartz, $L^{p}$ estimates for integral transforms, Trans. Amer. Math. Soc. 136 (1969), 33-50. MR 38 \#2638.

11. T. Walsh, On $L^{p}$ estimates for integral transforms, Trans. Amer. Math. Soc. 155 (1971), 195-215.

12. A. Zygmund, Trigonometric series. Vols. I, II, 2nd ed., Cambridge Univ. Press, New York, 1959. MR 21 \#6498.

Rutgers University, New Brunswick, New Jersey 08903 